\title{
HARNESSING CARDIAC ENERGETICS IN THE TREATMENT OF HEART FAILURE
}

\author{
Akshyay Pradhan
}

\section{ABSTRACT:}

Background: The prevalence of heart failure in India is not clearly known, in developed societies it is about 1 per cent of the elderly population (above 65 years) and is responsible for 20 per cent of all hospital admissions in this age group. Clinical trials that included ACEI, ARBs, beta-blockers and aldosterone antagonists have demonstrated substantial reduction in mortality in patients with systolic heart failure, but still the mortality remains high.

Aim: To recognize the altered energetics that play an important role in the mechanism of heart failure, role of cardiac metabolism and metabolic agents such as trimetazidine in the treatment of heart failure.

Results: Progression of heart failure leads to metabolic changes causing change in relative substrate utilization of fatty acids (60 to 90\%) and glucose (10 to 40\%) leading to a substantial reduction of ATP synthesis in the failing myocardium. Trimetazidine which is the most studied among the metabolic agents has shown to prefer glucose oxidation to fatty acid, significantly improving ATP synthesis by $+33 \%$. Randomized trials of trimetazidine have shown improvement in ejection fraction, $7-23 \%$, NYHA class, hospitalization and mortality reduction by $30 \%$.

Conclusion: Metabolic management offers fresh insights and among the metabolic agents available trimetazidine is the most documented. Trimetazidine has shown to improve cardiac function without affecting the heart rate or blood pressure. The mortality benefit and improvement in hospitalization frequency suggested need to be confirmed in larger randomized studies.

Key words: Cardiomyopathy, Cardiac metabolism, Metabolic agents, Trimetazidine

\section{INTRODUCTION}

Pericardial effusion (PE) associated with myocardial infarction (MI) is considered to be associated with anterior ST-segment elevation MI (STEMI), with large infarcts, and when heart failure is present. When PE is associated with cardiac tamponade, it is usually due to

Article received on 10 JULY 2016, published on 31 OCT 2016.

Akshyay Pradhan ${ }^{1}$,

Assistant Professor, Department of Cardiology, King George Medical University, Lucknow, Uttar Pradesh, India- 226003

Corresponding author: Akshyay Pradhan

Email: drakshyaypradhan@gmail.com, akshyaya33@grnail.com cardiac rupture, hemorrhagic pericarditis, or aortic dissection involving the right coronary artery [1]. However, the majority of PE does not cause hemodynamic compromise, and a small to moderate PE may often be viewed as a benign reaction to the MI. In a large study in the modern era of reperfusion from 25 French hospitals including 908 patients with MI, a PE was detected in only $6.6 \%$ of the patients [2].Patients had an echocardiogram performed at admission and again at discharge, and $0.8 \%$ developed left ventricular free wall rupture (FWR). This incidence of FWR is similar to that of a larger registry that did not search meticulously for rupture [3].

Acute FWR is one of the most common causes of mortality in acute MI. The diagnosis is most often made by the presence of sudden electromechanical dissociation followed by death if left untreated. Some patients survive for several hours, allowing time for The clinical syndrome of heart failure is the final pathway for several diseases that affect the heart. Although its prevalence in India is not clearly known, in developed societies, it is about 1 per cent of the elderly population (above 65 years). After showing a 159 per cent increase over the past decade, heart failure is now responsible for 20 per cent of all hospital admissions in this age group [1].

Over the years, numerous clinical trials with diuretics, vasodilators, inotropic agents, ACEIs, angiotensin receptor blockers, beta-blockers, and aldosterone antagonists have demonstrated substantial reduction in mortality of patients with systolic heart failure. Despite this, mortality remains high. After diagnosis, about 3040 per cent die within the first year, and $60-70$ per cent within 5 years. Thus for a person who survives up to 40 years of age, the risk is one in five of developing heart failure, and one in three of dying because of it within 1 year [2].

There is therefore a need for newer treatments that are effective and do not interfere with co-morbid disease, or interact with other drugs. The available evidence suggests that altered energetics play an important role in the mechanisms of heart failure [3]. For this reason, the modulation of cardiac metabolism has the potential to benefit patients with heart failure. Recent studies with 
agents that have a metabolic mode of action support the usefulness of such treatment.

\section{Pathophysiology of heart failure and current treatments}

The commonest underlying problem in heart failure is ventricular dysfunction due to an abnormal cardiac structure, function, rhythm, or conduction [2]. The usual cause is myocardial infarction, and hypertension. Of the several pathogenic theories that have been suggested, the hemodynamic model stressed the effect of altering load on the failing ventricle, and ushered in the era of diuretics, vasodilators and inotropic agents. The neurohumoral model recognised the importance of the renin-angiotensin-aldosteroneaxis and the sympathetic nervous system, leading to the use of ACEIs, angiotensin receptor blockers, beta-blockers, and aldosterone antagonists. Other studies have examined myocytes from failing hearts in an attempt to detect abnormal signaling, gene expression, or contractile protein structure. At present, a complex blend of structural, functional, and biologic alterations are evoked to explain the pathogenesis and progression of heart failure. Since no single pathophysiological model accounts for the varied clinical expressions, current therapy utilises several drugs, with each targeting one organ system [1].

\section{Cardiac energy metabolism}

Cardiac energy metabolism [4] has three main aspects. The first is the absorption and initial metabolism of food substrates. In this process there is cellular uptake of free fatty acids (FFA) and glucose; the breakdown of FFA by beta-oxidation and glucose by glycolysis; and the entry of the resulting intermediary metabolites into the Krebs cycle (Figure 1).

The second component is oxidative phosphorylation by which energy is produced by the mitochondrial respiratory chain. The phosphorylation of adenosine-diphosphate (ADP) by this mechanism produces the highenergy phosphate compound adenosine-tri-phosphate (ATP), which is the direct source of energy for the heart.

The third component is transfer of ATP generated within the mitochondria to the cell by a mechanism termed the creatine kinase energy shuttle (Figure 2). In this process, mitochondrial creatine kinase catalyses the transfer of the high-energy phosphate bond in ATP to creatine to form phosphocreatine and ADP. Phosphocreatine, a smaller molecule than ATP, rapidly diffuses out of the mitochondria to the myofibrils, where myofibrillar creatine kinase catalyses the reformation of ATP from phosphocreatine. This is then used by the myofibrils for contractile work and by the various pumps that maintain cellular homeostasis. The free creatine, formed by the removal of phosphate from phosphocreatine, diffuses back to the mitochondria. An important function of the creatine kinase system is to act as an energy buffer. When the energy demand out-strips the energy supply, the phosphocreatine level falls, keeping ATP at a normal level, but the free ADP level rises. The increased level of free ADP inhibits the function of many intracellular enzymes, causing failure of the heart's contractile mechanism. Thus, a metabolic derangement in the cardiac cell can occur when phosphocreatine levels fall and free ADP levels rise, even if ATP levels remain unchanged.

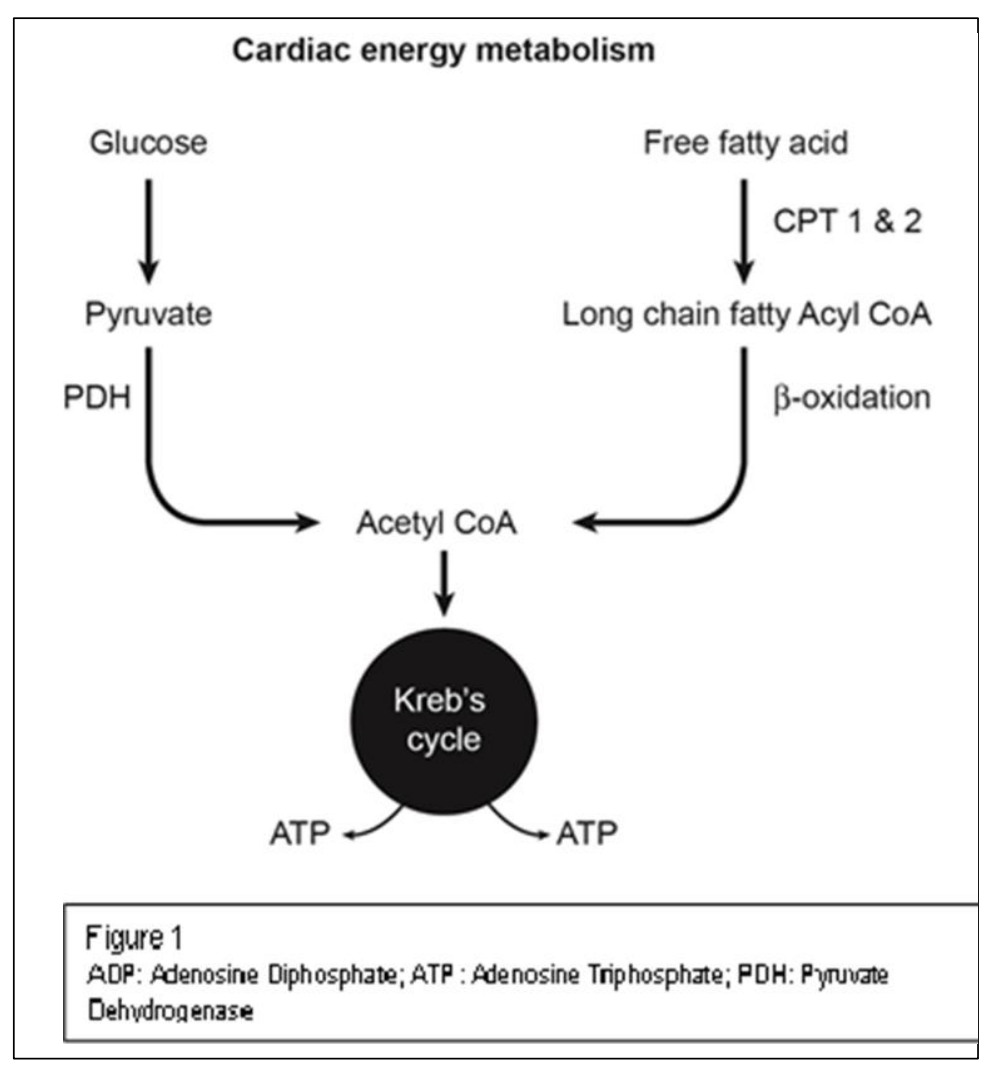

Metabolic changes in heart failure 
The initial response of the heart to injury is an increased sympathetic drive. This increases the contractile force of viable myocardium in an attempt to preserve and maintain normal cardiac output. The immediate metabolic effect is therefore a greatly increased expenditure and requirement of energy [6].

As failure progresses, changes in substrate utilisation can occur due to reduced uptake and reduced oxidation. There may also be a change in the relative contributions of fatty acids (60 to $90 \%$ ) and glucose (10 to $40 \%$ ) to ATP synthesis. Most studies suggest that fatty acid utilisation decreases; glucose utilisation increases in early heart failure, but because of insulin resistance in the myocardium, it decreases as failure progresses. However, there are simultaneous increases in plasma free fatty acids, glucose, and insulin concentrations. These increases make it difficult to interpret the metabolic changes within the heart muscle separately from the indirect influence of changes in the outside metabolic milieu.
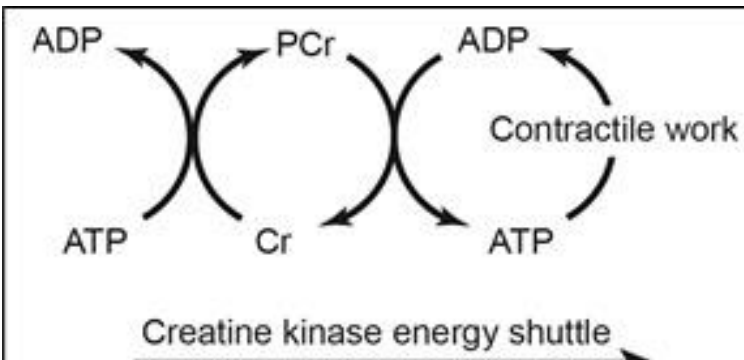

Figure 2

ADP: Adenosine Diphosphate; ATP: Adenosine Triphosphate;

$\mathrm{PCr}$ : Phospho creatine; $\mathrm{Cr}$ : Creatine

An impaired oxidative phosphorylation results in a substantial reduction of oxygen consumption and energy production in the failing myocardium. These is because cardiac mitochondria have structural abnormalities and are increased in number; the activity of electron transport-chain complexes and ATP synthase capacity are reduced; the regulation of oxidative phosphorylation by the phosphate acceptor ADP and creatine is impaired; and the levels of uncoupling proteins (which cause mitochondria to produce heat rather than ATP) may be increased.

An impaired creatinine kinase transfer system can lead to adecrease in ATP concentration; reduction in its transfer from the mitochondria to myofibrils; or an increase in ADP concentration. These changes can compromise contractile function.

Although myocardial ATP level remains normal during the early stages, it decreases by $30-40 \%$ when the failure is severe. The average ATP levels remain far above those required for ATP- consuming reactions and do not limit contractile function in heart failure. However, both phosphocreatine and total creatine levels decrease early and to a greater extent (by 30 to $70 \%$ ). Because of this down-regulation of the creatine transporter function, mitochondrial creatine kinase activity may decline to $20 \%$, and myofibril creatine kinase activity to $50 \%$, of normal values. This can lead to a $71 \%$ decrease in energy delivery to the myofibrils, and contractile dysfunction that is reflected in the loss of inotropic reserve.

During high-workload conditions, free ADP concentration doubles, further limiting contractile reserve that is manifested clinically as dyspnea on exertion.

The ratio of phosphocreatine to ATP is a powerful index of the energetic state of the heart. The creatine kinase reaction equilibrium favours ATP synthesis a 100 foldmorethanthe synthesis of phosphocreatine. Therefore, whenever the demand for ATP outstrips ATP synthesis, phosphocreatine levels decline first, and ATP decreases only when phosphocreatineis substantially depleted. In chronicheartfailure, however, total creatine level also falls, and this further decreases the phosphocreatine / ATP ratio.

Diabetes subjects are characterized by increased fatty acid oxidation and decreased glucose oxidation rates resulting in substrate utilization preference to fatty acids as energy source. Excessive fatty acid oxidation contributes to the energy metabolism and cardiac function abnormalities in diabetic cardiomyopathy. The accumulation of fatty acids in the heart as well as high rates of fatty acid oxidation leads to the development of diabetic cardiomyopathy [7].

\section{Deleterious effects of increased fatty acid oxidation:}

$\mathrm{NADH}$ and acetyl CoA produced by fatty acid oxidation in hibits the enzyme pyruvatede hydrogenase (PDH), thus decreasing the glucose metabolism. The increased fatty acid oxidation decreases cardiac efficiency up to $30 \%$ along with other mechanisms that are involved in the reduction of cardiac efficiency. Shifting the energy substrate to fatty acids can also result in mitochondrial 
reduced oxidative metabolism and increased glycolysis. The high glycolysis and low glucose oxidation rates can lead to proton production, resulting in alteration of ionic homeostasis and rerouting of ATP synthesized, away from contractile function towards re-instating the ionic homeostasis, thus decreasing cardiac efficiency. In diabetic cardiomyopathy fatty acid oxidation rates are elevated that contributes to impaired energy metabolism and cardiac function [7].

These abnormalities in cardiac energy metabolism occur early and are an integral part of the primary heart muscle disease. This suggests that the compromise of myocardial energetics has a causal role in heart failure. Therefore, the prevention of the semetabolic changes, common to all types of heart failure, without influencing hemodynamic factors orther ennin-angiotensin system forms the rationale for the use of metabolic agents in the treatment of heart failure [6].

\section{Metabolic agents in heart failure}

The drugs that are presently available for metabolic management act by modulating substrate utilisation. These compounds increase energy availability by partially reducing oxidation of fatty acids. Agents such as perhexiline and etomoxirsuppress the enzyme carnitinepalmitoyl transferase-1 that influences the uptake of plasma FFA and their transport to the mitochondria of cardiomyocytes. Other drugs such as trimetazidine directly inhibit FFA oxidation and indirectly promote glucose oxidation [8] Figure 3.

Trimetazidine, the first and most studied metabolic agent is widely used in clinical practice for the control of stable angina. It acts within the mitochondria of cardiomyocytes to suppress 3-ketoacyl-CoA, a key enzyme in the beta-oxidation of FFA. The suppression of fatty acid oxidation increases oxidative glycolysis. This shift in substrate utilisation yields more molecules of (ATP) and phosphocreatine. There is therefore an increase in overall energy generation with trimetazidine [9]. A randomized study recently demonstrated that in comparison to placebo, the addition of trimetazidine for 90 days to the treatment of patients with heart failure, significantly improved phosphocreatine / ATP ratio by as much as $33 \%$ [10].

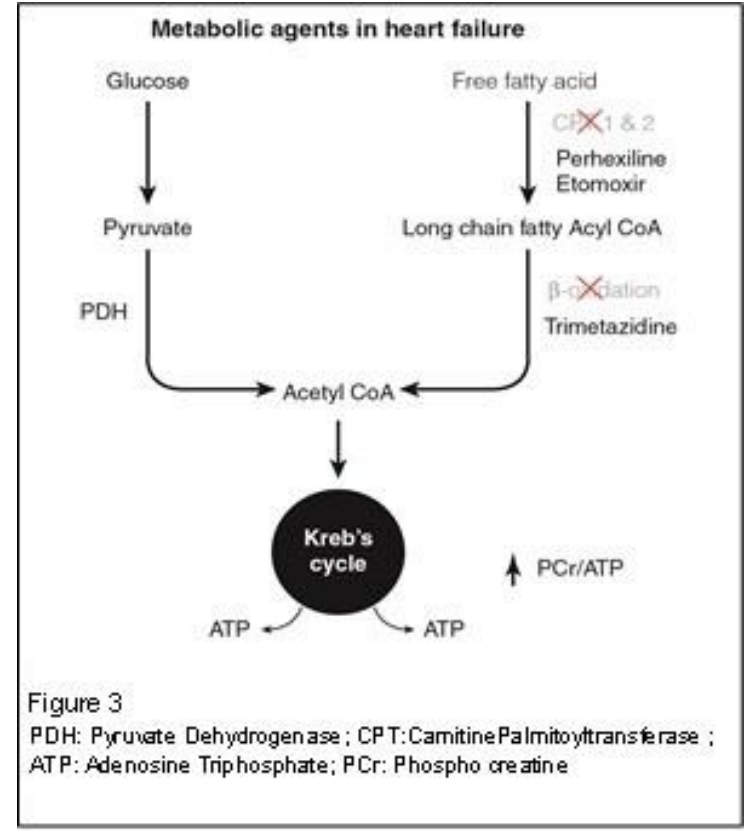

More than 15 years ago, a randomized trial on patients with NYHA class III or IV ischemic cardiomyopathy demonstrated that in comparison to placebo, the addition of trimetazidine to usual treatment for 6 months, significantly increased ejection fraction by $9.3 \%$ vs a decrease of $15.6 \%$ with placebo, while cardiac volume decreased by $7 \%$ vs $4 \%$. Both angina and dyspnea showed significant improvement with trimetazidine [11].

More recently, a study on similar patients using low dose dobutamine echocardiography coupled with an exercise test showed that in comparison to placebo, trimetazidine significantly increased ejection fraction by $19.7 \%$, peak oxygen volume by $15 \%$, and improved wall thickening score index at rest and peak exercise by $13 \%$ and $20.7 \%$ respectively [12]. In another randomized placebo controlled cross over study on patients with coronary artery disease with a positive dobutamine stress test, in comparison to the placebo response, trimetazidine significantly lowered to a greater extent wall motion score index at rest by 0.06 and at peak dobutamine infusion by 0.1 . Further, dobutamine infusion time was greater by 2.6 minutes and its dose by $6 \mathrm{mcg} / \mathrm{Kg} / \mathrm{min}$ in patients treated with trimetazidine [13]. The usefulness of trimetazidine in the management of early heart failure over the medium term was shown in a randomized study of 6 months duration on elderly patients with left ventricular dysfunction. In comparison to placebo, there was significantly greater increase in left ventricular ejection fraction by $7 \%$, together with a 
significantly greater $5 \mathrm{~mm}$ decrease in left ventricular end diastolic and systolic diameter with trimetazidine. Quality of life scores showed significant improvement versus no change with placebo [14].

In two randomized studies over the long term, addition of trimetazidine to standard treatment of patients with dilated ischemic cardiomyopathy for 18 months significantly improved NYHA class and left ventricular function. Ejection fraction increased by $7 \%$ with a 20 $\mathrm{ml} / \mathrm{msq}$ decrease in left ventricular end diastolic volume, compared to a worsening of both parameters with standard treatment alone. In addition, the inflammatory process assessed by plasma $\mathrm{C}$ reactive protein concentration, progressed in patients on standard treatment but remained stable in those receiving trimetazidine [15].

These benefits have been confirmed in 200 patients with multi-vessel coronary artery disease and left ventricular dysfunction. Patients randomized to receive trimetazidine or placebo in addition to standard treatment were assessed at baseline and after 24 months with a resting gated SPECT imaging test, followed by an exercise test and post exercise injection of radioactive isotope with imaging by gated SPECT. In addition to functional improvement, there was a significant $89 \%$ increase in the systolic wall thickness score, with a $23 \%$ increase in ejection fraction. An important result of this study was that at the end of 24 months treatment, $92 \%$ of patients receiving trimetazidine versus $62 \%$ receiving placebo survived [16]. This suggests that the addition of Trimetazidine to standard treatment may confer a $30 \%$ mortality benefit in heart failure over the long term.

The long-term benefit of adding trimetazidine to the usual treatment of patients with non- ischemic heart failure has also been recently established. In an open label study, 55 patients with non-ischemic dilated cardiomyopathy under conventional treatment (that included ACEI/ARB in all, and B-blockers in 75\%) were randomized to conventional treatment plus trimetazidine or conventional treatment alone. After a mean 13 month follow up, clinical assessment, exercise testing and two-dimensional echocardiography demonstrated that the addition of trimetazidine significantly improved NYHA functional class, together with a significant $7 \%$ decrease in left ventricular endsystolic volume, and a $7 \%$ increase in ejection fraction. By contrast, conventional treatment alone without trimetazidine was associated with an increase in both left ventricular end-systolic and diastolic volumes while ejection fraction significantly decreased [17].

A recent international multicenter retrospective study by Fragasso et al. [21] included 669 heart failure patients predominantly of ischemic origin with ejection fraction of $<45 \%$, NYHA class II to IV who were optimally treated with conventional therapy including RAAS inhibitors, beta-blockers and diuretics for an average follow-up of 38 months. Addition of trimetazidine demonstrated a significant benefit in improving global survival by $11.3 \%$, CV death by $8.5 \%$ and the rate of hospitalization for cardiovascular causes was reduced by $10.4 \%$ compared to patients who were not on trimetazidine.

In a meta-analysis by Zhang et al. [22] involving 884 heart failure patients, addition of trimetazidine was associated with significant improvement in LVEF by $6.5 \%$, exercise time by 64 seconds, NYHA class by 0.57 , BNP levels and reduction in hospitalization for cardiac causes (RR 0.43, $\mathrm{p}=0.03$ ).

Grajek et al [23] in their recent meta-analysis confirmed similar benefits in heart failure patients with the addition of trimetazidine with a significant reduction in all-cause mortality $(R R=0.28, p<0.0001)$

\section{Efficacy in female patient population}

Many a time female populations are underrepresented in clinical trials though the incidence is as much as in male population. Trimetazidine is equally effective in male and female patients. In the meta-analysis of Zhang et al.[22] an observation of female heart failure patients were more likely to be benefited from trimetazidine treatment regarding left ventricular ejection fraction. But this needs to be evaluated and confirmed in a bigger prospective study.

The recent consensus statement on the management of chronic heart failure in India quotes, that the data on trimetazidine is promising and often used in patients with heart failure [24]. The more recent 2016 ESC guidelines for the diagnosis and treatment of acute and chronic heart failure, recommend treatment with trimetazidine for heart failure patients with comorbidities such as angina with Level of evidence A and class IIb for the treatment of stable angina patients with symptomatic (NYHA Class II-IV) heart failure with reduced ejection fraction [25]. 
In all these studies the effect of trimetazidine on blood pressure and heart rate was similar to placebo, and there was no adverse effect on co-morbid disease or interaction with other treatments.

\section{Other metabolic agents:}

Perhexiline is an inhibitor of Carnitine palmitoyltransferease-1 (CPT-1), thus inhibiting the entry of medium and long chain fatty acids into mitochondria and subsequently reducing the fatty acid oxidation and relative increase in glucose oxidation. A randomized controlled study on 56 patients assessed the addition of Perhexiline to standard treatment versus placebo for 2 months [18], which demonstrated an increase in LVEF. A more recent study involving 50 heart failure study of non-ischemic etiology, addition of perhexiline $200 \mathrm{mg}$ for one duration significantly improved the phosphocreatine to ATP ratio (1.16 to 1.51, $\mathrm{p}<0.001)$ and improvement in NYHA functional class $(\mathrm{p}=0.036)$. However perhexiline treatment did not improve LVEF and there was no difference in substrate utilization compared to placebo [19].

Etomoxir, also an inhibitor of CPT, in an uncontrolled study on 10 patients assessed the addition to standard treatment for 3 months [20].Etomoxir improved the left ventricular ejection fraction. However, the results of these small studies require confirmation in large randomized clinical trials. Due to severe hepatotoxicity the clinical development of etomoxir has been terminated.

Inhibitors of malonyl CoA (MCD) has shown to increase glucose oxidation, reduce fatty acid oxidation and improve insulin sensitivity [26, 27, 28]. But the role of MCD inhibitors are yet to be established in heart failure. PPAR (peroxisome proliferative-activated receptor) drugs such as thiazolidinediones (TZDs) and fibrates were thought to have beneficial effects and increase cardiac efficiency. But diabetic patients treated with pioglitazone a PPAR $\gamma$ in the PROACTIVE study showed an increased incidence of heart failure [29]. Fibrates from PPAR $\alpha$ family have shown mixed results with no reduction on cardiac mortality while PPARS has shown to prevent cardiomyocyte hypertrophy and increase in glucose oxidation.

Ranolazine a late $\mathrm{Na}+$ current (INa) inhibitor has been tested in a small proof-of-concept study of 20 patients with diastolic heart failure with no improvement in echocardiographic, cardiopulmonary exercise and NTpro-BNP levels (30).In another study involving a combibation of systolic and diastolic heart failure patients, treatment with ranolazine $1000 \mathrm{mg}$ twice daily significantly improved LVEF ( $30.46 \%$ to $36.83 \%$, $\mathrm{p}=0.018$ ) in systolic heart failure patients and $42.83 \%$ to $52.33 \%$, $\mathrm{p}=0.002$ ) in diastolic heart failure patients [31].

SGLT2 (sodium-glucose co-transporter-2) inhibitors are the compounds which inhibit the sodium glucose cotransporter-2 and inhibits the reabsorption of glucose at the proximal convoluted tubule in the kidneys and facilitate glucosuria thus lowering plasma glucose [32]. These agents also promote weight loss particularly reducing the total body fat. Dapagliflozin and empagliflozin have shown to favor fatty acid metabolism to glucose metabolism. Dapagliflozin increases fatty acid oxidation by $14 \%$ compared to $20 \%$ reduction in glucose oxidation. Similarly empagliflozin in a 4 week study has demonstrated to decrease glucose oxidation by $60 \%$ and increase fatty acid oxidation by $20 \%$ (33). The results of EMPA-REG OUTCOME study (34) with empagliflozin, reduced the primary composite endpoint (PEP) of CV death, non-fatal MI and non-fatal stroke by a significant $14 \%$ (HR 0.86, 95\% CI; $0.74-0.99$, $\mathrm{p}=0.04$ ) and hospitalization for heart failure by $35 \%$ (HR $0.65,95 \% \mathrm{CI} ; 0.50-0.85, \mathrm{p}=0.002)$. The results are particularly intriguing given the decrease in heart failure hospitalization in relation to increase in fatty acid oxidation versus reduced glucose oxidation with empagliflozin and is unlikely to be the reason for these beneficial effects on heart failure outcomes [33].

The shift of substrate utilization from free fatty acids to glucose has shown to provide some contrasting results. In an experimental study of male wistar rats with heart failure after myocardial infarction, fed with high saturated fat for 8 weeks improved myocardial contractile function at rest and during physiological stress, but negatively impacted the contractile reserve under non pathological conditions [35]. Data from Tuunanen et al has demonstrated that acute depletion of free fatty acid in patients with idiopathic dilated cardiomyopathy compared with healthy volunteers, FFA uptake decreased by $>80 \%$ in both groups and resulted in reduced cardiac work and efficiency [36]. It's important to note that these results are based on small sample of patients in acute situation and patients of nonischemic cardiomyopathy. Chronic depletion of FFA in other forms of HF needs to be explored. 


\section{Conclusion}

Treating heart failure is a challenge to the physician because of its rapidly rising incidence, high rates of hospitalization and mortality, complexity due to comorbid diseases, and interactions between many of the drugs that are used. Against this background, fresh insights into the metabolic derangements that accompany heart failure have opened a new avenue for its management.

Of the metabolic agents, trimetazidine is the most studied. It causes a shift in substrate metabolism to increase energy generation. In patients with heart failure, Itis effective in improving cardiac function without interfering with associated disease, changing heart rate and blood pressure, or interacting with other drugs. Although a mortality benefit and improvement in frequency of hospitalisation has been suggested, these end points need confirmation in larger randomized studies. The evidence so far suggests that combining trimetazidine with existing treatments can help improve the outcome of heart failure.

\section{References}

1. Jessup M, Brozena S, Heart Failure. N Engl J Med 2003;348:2007-18.

2. McMurray JJV, Pfeffer MA. Heart failure. Lancet 2005; 365: 1877-89.

3. Mann DL, Bristow MR. Mechanisms and models in heart failure: the biomechanical model and beyond. Circulation 2005;111:2837-49. [Erratum, Circulation 2005;112(4):e75].

4. Neubauer S. The Failing Heart - An Engine Out of Fuel. N Engl J Med 2007;356:1140-51.

5. Stanley WC, Recchia FA, Lopaschuk GD. Myocardial substrate metabolism in the normal and failing heart. Physiol Rev 2005;85:1093-129.

6. Packer M. Pathophysiology of chronic heart failure. Lancet 1992;340:88-92

7. Fillmore N, Mori J, Lopaschuck GD. Mitochondrial fatty acid oxidation alterations in heart failure, ischaemic heart disease and diabetic cardiomyopathy. Bri J Pharmacol 2014;171:20802090.

8. Opie LH. The metabolic vicious cycle in heart failure. Lancet 2004; 364:1733-4.

9. Lopaschuk GD. Pharmacologic rationale for trimetazidine in the treatment of ischemic heart disease. Am J Cardivasc Drugs 2003; 3 Suppl,1:21-6.

10. Fragasso G, Perseghin G, De Cobell F, et al. Effects of metabolic modulation by trimetazidine on left ventricular function and phosphocreatine/adenosine triphosphate ratio in patients with heart failure. European Heart Journal 2006;27:942-948.

11. Brottier L, Barat JL, Combe C, Boussens B, Bonnet J, Bricaud $\mathrm{H}$. Therapeutic value of a cardioprotectiv e agent in patients with severe ischemic cardiomyopathy. Eur Heart J 1990;11:207-12.

12. Beardinelli R, Purcaro A. Effects of trimetazidine on the contractile response of chronically dysfunctional myocardium to low dose dobutamine in ischemic cardiomypathy. Eur Heart J 2001;22:2164-70.

13. Lu C, Dabrowski P, Fragasso G, Chierchia SL. Effects of Trimetazidine on ischemic left ventricular dysfunction in patients with coronary artery disease. Am J Cardiol 1998;82:898-901.

14. Vitale C, Wajngaten M, Sposato B, et al. Trimetazidine improves left ventricular function and quality of life in elderly patients with coronary artery disease. European Heart Journal 2004;6:1-8.

15. Di Napoli P, Taccardi AA, Barsotti A. Long term cardioprotective action of trimetazidine and potential effect on the inflammatory process in patients with ischaemic dilated cardiomyopathy. Heart 2005;91:161-165.

16. El-Kady T, El-Sabban K, Gabali M, et al. Effects of trimetazidine on myocardial perfusion and the contractile response of chronically dysfunctional myocardium in ischemic cardiomyopathy. Am J Cardiovasc drugs 2006;5:271-8. 
17. Fragasso G, Palloshi A, Puccetti P, Silipigni C, Rossodivita A, Pala M, et al. A Randomized clinical trial of trimetazidine, a partial free fatty acid oxidation inhibitor, in patients with heart failure. J Am Coll Cardiol 2006; 48:992- 8.

18. Lee L, Campbell R, Scheuermann- Freestone M, et al. Metabolic modulation with perhexiline in chronic heart failure: a randomized, controlled trial of shortterm use of a novel treatment. Circulation 2005;112:3280-8.

19. Roger MB, Williams LK, Kuehl M, Bowater S, Abozguia K, Leyva F, Yousef Z, Wagenmakers AJM, Thies F, Horowitz J, Frenneaux MP. Improvement in cardiac energetics by perhexiline in heart failure due to dilated cardiomyopathy. JACC: Heart Failure 2015;3(3):202-11.

20. Schmidt-Schweda S, Holubarsch C. First clinical trial with etomoxir in patients with chronic congestive heart failure. Clin Sci (Lond) 2000;99:27-35.

21. Gabriele Fragasso, Giuseppe Rosano, et al. Effect of partial fatty acid oxidation inhibition with trimetazidine on mortality and morbidity in heart failure: Results from an international multicentre retrospective cohort study. Int J Cardio 2013;320-325.

22. Lei Zhang, Yizhou Lu, et al. Additional Use of Trimetazidine in Patients with Chronic Heart Failure. JACC 2012;59:913-922.

23. Stefan Grajek, Michal Michalak. The Effect of Trimetazidine Added to Pharmacological Treatment on All-Cause Mortality in Patients with Systolic Heart Failure. Cardiology 2015;131:22-29.

24. Seth S, Bhargava B, Maulik SK, McDonagh T, Saxena A, Airan B, Calambur N, Hote M, Parakh N, Bahl A, Ramakrishnan S, Chaturvedi V, Nath R, Chakraborthy P. Consensus statement on management of chronic heart failure in India. J PractCardiovascSci 2015;1:105-12.

25. The task force for the diagnosis and treatment of acute and chronic heart failure of the European society of Cardiology, 2016 ESC guidelines for the diagnosis and treatment of acute and chronic heart failure Eur Heart J 2016;37:2129-2200.

26. Dyck JR, Chen JF, Stanley WC, Barr R, Chandler MP, Brown S. Malonyl coenzyme a decarboxylase inhibition protects the ischemic heart by inhibiting fatty acid oxidation and stimulating glucose oxidation. Circulation research 2004;94:e78-84.

27. Stanley WC, Morgan EE, Huang H, McElfresh TA, Sterk JP, Okere IC. Malonyl-CoA decarboxylase inhibition suppresses fatty acid oxidation and reduces lactate production during demand-induced ischemia. Am J Physiol Heart CircPhysiol 2005;289:H2304-2309.

28. Cheng JF, Huang Y, Penuliar R, Nishimoto M, Liu L, Arrhensius T. Discovery of potent and orally available maolonyl-CoA decarboxylase inhibitors as cardioprotective agents. J Med Chem 2006;49:40554058 .

29. Dormandy JA, Charbonnel B, Eckland DJA, Erdmann E, Massi-Benedetti M, Moules IK. Secondary prevention of macrovascualr events in patients with type 2 diabetes in the PROactive study (PROspectivepioglitAzone Clinical Trial In macroVascular Events): a randomized controlled trial. Lancet 2005;366:1279-1289.

30. Maier LS, Layug B, Ewa KP, Belardinelli L, Lee S, Sander J, Lang C, Wachter R, Edelmann F, Hasenfuss G, Jacobshagen C. Ranolazine for the treatment of diastolic heart failure in patients with preserved ejection fraction: the RALI-DHF proof-ofconcept study. JACC Heart Failure 2013;1(2):115-22.

31. Murray GL, Colombo J. Ranolazine preserves and improves left ventricular ejection fraction and autonomic measures when added to guidelinedriven therapy in chronic heart failure. Heart Intl. 2014;9(2):66-73.

32. Kalra S. Sodium glucose co-transporter-2 inhibitors: A review of therir basic and clinical pharmacology. Diabetes Ther. 2014;5:355-366. 
33. Abdul-Ghani M, Del Prato S, Chilton R, DeFronzo RA. SGLT2 inhibitors and cardiovascular risk: Lessons learned from the EMPA-REG OUTCOME study. Diabetes Care 2016;39:717-725.

34. Zinman B, Wanner C, Lachin JM, Fitchett D, Blumhmki E, Hantel S, Mattheus M, Devins T, Johansen OE, Woerle HJ, Broedl UC, Inzucchi SE for the EMPA-REG OUTCOME investigators. Empagliflozin, cardiovascular outcomes, and mortality in type 2 diabetes. NEJM September 17, 2015.
35. Jessica MB, Molly SB, Tracy AM, Xiaoquin C, Salman A, Martin EY, Brian DH, Margaret PC. The myocardial contractile response to physiological stress improves with high saturated fat feeding in heart failure. Am J Physiol Heart CircPhysiol 299: H410-H421, 2010

36. Helena T, Erik E, Alexandru N, Kjell N, Birger H, Juhani KEA, Pirjo N, Patricia I, Heikki U, Opie LH, Juhani K. Free fatty acid depletion acutely decreases cardiac work and efficiency in cardiomyopathic heart failure. Circulation 2006;114:2130-2137 